\title{
Weibull Analysis of Electrical Breakdown Strength as an Effective Means of Evaluating Elastomer Thin Film Quality
}

Silau, Harald; Stabell, Nicolai Bogø; Petersen, Frederik Riddersholm; Pham, Martin; Yu, Liyun; Skov, Anne Ladegaard

Published in:

Advanced Engineering Materials

Link to article, DOI:

10.1002/adem.201800241

Publication date:

2018

Document Version

Early version, also known as pre-print

Link back to DTU Orbit

Citation (APA):

Silau, H., Stabell, N. B., Petersen, F. R., Pham, M., Yu, L., \& Skov, A. L. (2018). Weibull Analysis of Electrical Breakdown Strength as an Effective Means of Evaluating Elastomer Thin Film Quality. Advanced Engineering Materials, 20(9), [1800241]. https://doi.org/10.1002/adem.201800241

\section{General rights}

Copyright and moral rights for the publications made accessible in the public portal are retained by the authors and/or other copyright owners and it is a condition of accessing publications that users recognise and abide by the legal requirements associated with these rights.

- Users may download and print one copy of any publication from the public portal for the purpose of private study or research.

- You may not further distribute the material or use it for any profit-making activity or commercial gain

- You may freely distribute the URL identifying the publication in the public portal 
DOI: 10.1002/adem.((please add manuscript number))

\section{Weibull analysis of electrical breakdown strength as an effective means of evaluating elastomer thin film quality}

By Harald Silau, Nicolai Bog $\varnothing$ Stabell, Frederik Riddersholm Petersen, Martin Pham, Liyun Yu and Anne Ladegaard Skov*

[*] Prof. Dr. Anne Ladegaard Skov, Harald Silau, Nicolai Bogф Stabell, Frederik Riddersholm Petersen, Martin Pham, Dr. Liyun Yu Danish Polymer Centre, Department of Chemical and Biochemical Engineering, Technical University of Denmark, Søltofts Plads Building 227 Room 122

2800 Kgs. Lyngby, Denmark

Email:al@kt.dtu.dk

To realize the commercial potential of dielectric elastomers, reliable, large-scale film production is required. Ensuring proper mixing and subsequently avoiding demixing after e.g. pumping and coating of elastomer premix in an online process is not facile. Weibull analysis of the electrical breakdown strength of dielectric elastomer films is shown to be an effective means of evaluating the film quality. The analysis is shown to be capable of distinguishing between proper and improper mixing schemes where similar analysis of ultimate mechanical properties fails to distinguish.

\section{Introduction}

Dielectric elastomers are finding more and more applications, but the production of these thin elastomeric films is complex and remains a challenge. The production of thin, large area, and inherently soft elastomers poses a great challenge to the final product reliabilit, ${ }^{[1,2]}$ because thickness variations as well as local variations in elastomer composition greatly affect the overall properties of the resulting film and thus of the transducer. Currently, poor film quality as a result of improper mixing is usually realised in retrospect when a film fails. This is a great challenge in the production since mixing of the thermosetting elastomer must be done 
fast and efficiently without excessive heating in order not to cure the elastomer during the mixing process.

The precise measurement of the thickness of elastomer films is of high importance as well and these online measurements are well-known from solar cell production etc. ${ }^{[3,4]}$ Optical techniques are generally preferable due to their non-contact nature for avoiding deformations of the inherently soft films. Different optical methods can be used, such as white light interferometry, laser profilometry, and transmission or reflection spectrometry. ${ }^{[5]}$ Commonly for large-scale production of dielectric elastomers, thicknesses of 20 micron are desired, but novel techniques allow for the production of even thinner films, even submicron, ${ }^{[6-8]}$ where sensitivity to thickness variations is even greater.

Because the actuation strain of dielectric film scales with the thickness to the second power, ${ }^{[9]}$ dielectric elastomers are very sensitive to variations in thicknesses, with local disparities causing severe local thinning commonly followed by electrical breakdown through so-called 'electromechanical instability'. ${ }^{[10]}$ However, depending on the electrode geometry, this phenomenon may be reduced. ${ }^{[11]}$ Variations in thickness after coating are inherent in nonideal elastomer mixtures and are the result of discrepancies in viscosities. Numerous additives in the formulations may overcome this issue, but they cannot compensate for improper mixing, which means that consistent and coherent mixing of the elastomer premixes is important for achieving homogeneous films, with respect to thickness, local homogeneity and the consistent presence of the reactants throughout the film. Due to the significant differences in molecular weights, and therefore also differences in viscosities of the crosslinker and the polymer (viscosity scaling with a molecular weight to the power of $\sim 3.4$ ), viscous components may be slightly immiscible in lower viscosity components. If a less viscous fluid is forced into a more viscous one, the interface between the two fluids may become unstable and long fingers of the less viscous fluid may arise as a quasi-stable 
condition. ${ }^{[12]}$ This phenomenon cannot be detected by traditional means such as NMR and IR spectroscopy, and thus an initial evaluation of mixing quality is not easy. Furthermore, mixing cannot be extended unlimitedly, since the elastomer premixes will start to react to lesser or greater extent and thereby further complicate the evaluation if viscosity is used as a measure for homogeneity. Using a value of viscosity as a target value is also problematic, since a given viscosity may arise from many different mixture conditions, i.e. it is not by any means a singular measure; therefore, the evaluation naturally can be considered after coating. Film quality is a result of mixing, coating and curing processes, though it also depends on the specific elastomer formulation, which may be formulated with additives to provide a smoother surface, longer pot life, less sensitivity to curing inhibition, etc. ${ }^{[13]}$ Improper mixing leads to inhomogeneity in the reactants, as discussed previously, but by introducing the controlled heterogeneity of silicone elastomer films, mechanical properties can be altered very effectively if the crosslinking concentration is increased locally in microscopic "spots" and thus lowered in the remaining matrix. ${ }^{[14]}$ This phenomenon leads to a strong decrease in the Young's modulus and a simultaneously higher ultimate strength due to the reinforcing effect of the densely crosslinked spots. The same effect can result in improper mixing of the network constituents and may be ideal, for example, in soft actuation where a low Young's modulus is desirable; on the other hand, in this case, reproducibility is not existent. Additionally, a surplus of solvent may lead to the loss of entanglements and thus a strong softening effect. ${ }^{[15]}$ Detecting local nano-structural or microstructural variations, however, is not easy but can be performed by $\mathrm{AFM}^{[16]}$ or nanoindentation, ${ }^{[17]}$ though this requires access to advanced instrumentation.

Ultimate mechanical properties such as tensile strength are very sensitive to minute imperfections, and usually they are governed by the largest imperfection, not the degree thereof. Thus, they usually do not give an overall picture of the film quality but rather the 
number of crucial imperfections in that particular filmstrip (which may be none for a certain strip and multiple for another).

Variations in crosslinking are also evident from changes in electrical properties. ${ }^{[18]}$ For thin film evaluation, the goal is to carry out as few tests as possible while reliability is consistently determined. Electrical breakdown strength has been found to be a good indicator of film quality, and therefore further statistical analyses in this regard will potentially be beneficial. Electrical breakdown strength is determined as the maximum applied electric field over a given film before short-circuiting takes place. Various methods to determine electrical breakdown strength of DEs have been reported in literature. ${ }^{[19,20]}$ In the utilized method within this study the geometrical parameters of the electrodes have been kept constant such that the contacted area remains constant. Likewise the ramp-up of the electrical field has been kept constant during the experiments to eliminate the influence of build-up of charges.

One method to describe the statistical probability of the electrical breakdown of an elastomer in a given electrical field is the Weibull distribution. The usual Weibull distribution is given by three parameters, namely $\eta$, which describes the characteristic life, $\beta$, which is the shape parameter, and $\gamma$, which is the location parameter. ${ }^{[21]} t$ is the variable, which in this case is the electrical field over the dielectric elastomer. The probability density function (pdf) is described by:

$$
f(t)=\frac{\beta}{\eta}\left(\frac{t-\gamma}{\eta}\right)^{\beta-1} e^{-\left(\frac{t-\gamma}{\eta}\right)^{\beta}}
$$

In most cases, the location parameter is set to $\gamma=0$, which gives the two-parameter Weibull distribution:

$$
f(t)=\frac{\beta}{\eta}\left(\frac{t}{\eta}\right)^{\beta-1} e^{-\left(\frac{t}{\eta}\right)^{\beta}}
$$

The value of the location parameter of 0 indicates that electrical breakdown may occur instantaneously with the application of an electrical field, i.e. $f(t)>0$ for $t>0$. If the location 
parameter is given by $\gamma=\gamma^{\prime}$, then $\mathrm{f}(\mathrm{t})>0$ for $\mathrm{t}>\gamma^{\prime}$. The approximation of $\gamma=0$ seems reasonable for the electrical breakdown of dielectric elastomer films, since a conducting fibre/dust particle within the elastomer, for example, may lead to immediate breakdown upon the application of an electrical field.

The above distribution can also be expressed as a cumulative density function (cdf) by the integration of the pdf:

$$
F(t)=1-e^{-\left(-\frac{t}{\eta}\right)^{\beta}}
$$

where, in other words, $\eta$ is electrical breakdown strength and $\beta$ is a measure of reliability. Usually, $\eta$ agrees with the arithmetic mean within a few $V / \mu \mathrm{m} \cdot{ }^{[22]}$ An illustration of how the two Weibull parameters, $\eta$ and $\beta$, affect the probability density and cumulative density functions is shown in Figure 1.
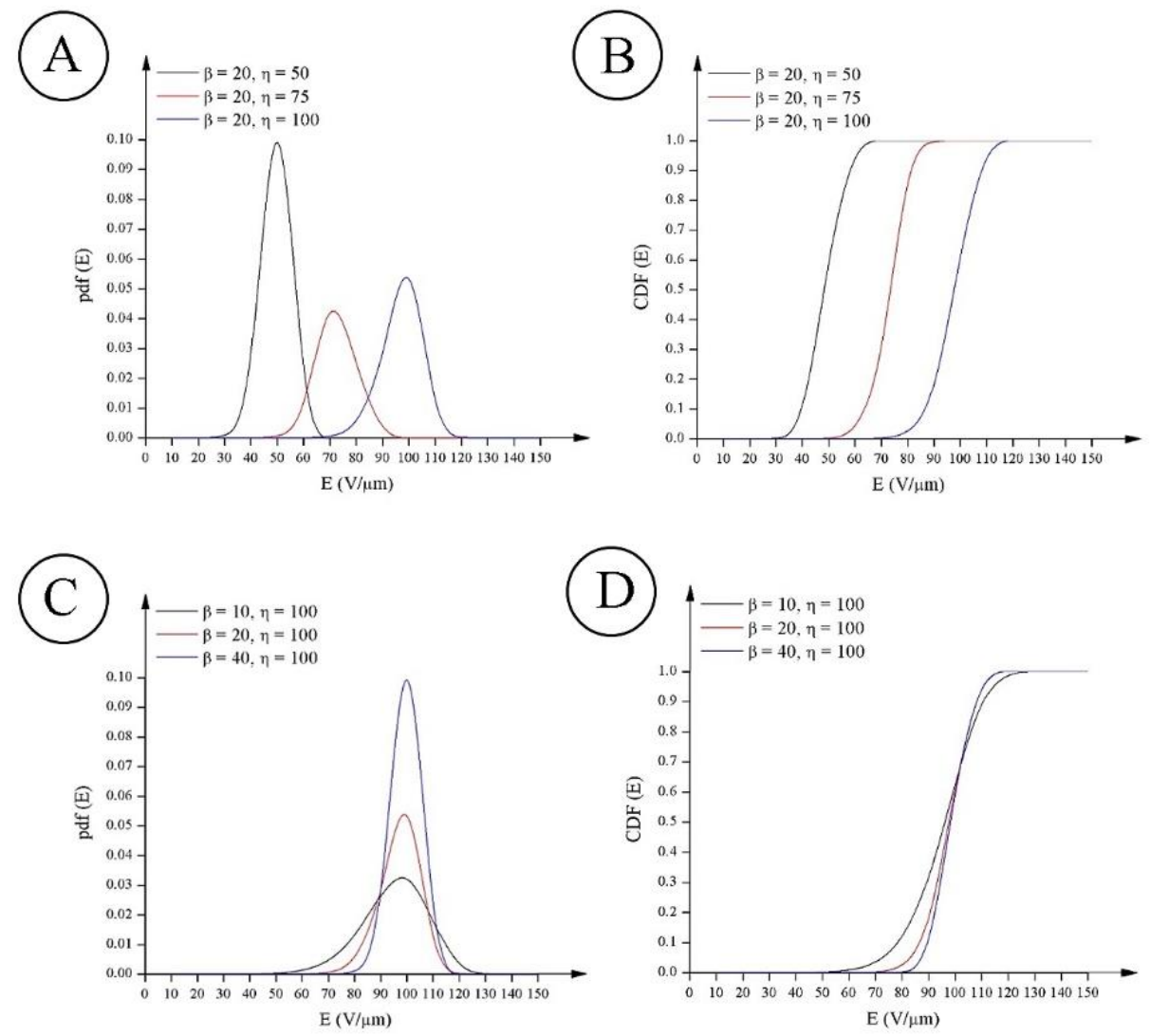

Fig.1. Illustration of the effect of $\eta$ and $\beta$ on the $p d f(l e f t)$ and cdf (right) of the Weibull distribution. A) and B) illustrate how variations in $\eta$ at constant $\beta$ affect the Weibull 
distribution, and C) and D) illustrate how variations in $\beta$ at constant $\eta$ affect the Weibull distribution.

When producing dielectric elastomers in the laboratory by means of blade coating, the resulting thickness of a given film is dependent - obviously - not only on the utilised gap, but also on the viscosity of the resulting mixture and thus on the time from and temperature during mixing and coating etc. To reduce the amount of tests needed to determine if the elastomers are reliable, extrapolation of the breakdown strength, $E_{0}$, to different thicknesses can be achieved. As proposed by Zakaria et al., this can be done with the following theoretical expression: ${ }^{[20]}$

$$
\begin{aligned}
& \left(\frac{E_{n}}{\eta}\right)^{\beta}=n\left(\frac{E_{0}}{\eta}\right)^{\beta} \\
& E_{n}=n^{-\frac{1}{\beta}} E_{0}<E_{0}
\end{aligned}
$$

where $n$ is a factor describing the increase in the elastomer thickness, in which case it is given that $n>1$. For this expression to be valid for different thicknesses, the shape parameter, $\beta$, has to be constant for all given thicknesses, though this is not a generally valid approximation for dielectric elastomers. ${ }^{[23]}$

The value of the shape parameter (width of distribution) depends on how uniform the breakdown strength is throughout the elastomer, i.e. it depends on microscopic homogeneity. In the following, a given commercial elastomer will be tested for both its dielectric breakdown strength and its stress strain characteristics, to determine whether the shape parameter is constant or it varies with elastomer thickness, and if there is a connection between mechanical properties and the size of the shape parameter.

Current state-of-the-art techniques to increase the reliability of elastomer films include a socalled "pre-treatment" whereby the coated and cured elastomer film is taken stepwise to a given electrical field $\left(\mathrm{E}_{\mathrm{pre}}\right)$. As a result, all breakdowns occurring before $\mathrm{E}_{\text {pre }}$ are eliminated, 


\section{Experimental section}

\subsection{Materials}

A liquid silicone rubber (LSR), Elastosil LR3043/50 (LR3043/50), was supplied by Wacker Chemie AG, Germany. The solvent used for the samples was OS20, purchased from Dow Corning, USA. 


\subsection{Sample preparation}

Three separate batches of LR3043/50 were prepared by mixing component A, component B and the OS20 solvent in the ratio 5:5:7 by mass on different days (batch 1: day 1, batch 2: day 8, batch 3: day 15). Batch 3 premixes were exposed to the air for two weeks in the storage container, whereas premixes from batches 1 and 2 were kept in airtight containers flushed with nitrogen. Each batch mixture was split into two (at a ratio of 3:1), the largest one of which was speed-mixed at $3500 \mathrm{rpm}$ for $2 \times 3 \mathrm{~min}$, followed by $2 \times 5 \mathrm{~min}$ to get a homogeneous mixture, and the other was hand-mixed for $15 \mathrm{~min}$. From each batch, four films were made by applying the mixture to glass plates with a 3540 bird applicator (Elcometer). The three speed-mixed samples were applied with thicknesses of $50 \mu \mathrm{m}, 100 \mu \mathrm{m}$ and 150 $\mu \mathrm{m}$, respectively, and the hand-mixed sample was applied with a thickness of $100 \mu \mathrm{m}$. All films were cured at $45^{\circ} \mathrm{C}$ for 1 hour, followed by 30 minutes at $115^{\circ} \mathrm{C}$. This extensive postcuring scheme was utilised to ensure that all volatiles had been removed efficiently and therefore would not influence the results. ${ }^{[22,26]}$ The produced films and their thickness can be seen in Table 1.

Table 1. Final thicknesses of the elastomer films after curing. Each knife gap is specified in the second row, and the true film thicknesses of different batches for various samples are subsequently stated. The films are coated in a consecutive manner.

Final thickness $[\mu \mathrm{m}]$

Batch No. Applied knife gap [ $\mu \mathrm{m}]$

\begin{tabular}{lllll} 
& 50 (speed-mix) & 100 (speed-mix) & 150 (speed-mix) & 100 (hand-mix) \\
\hline 1 & $31 \pm 1$ & $46 \pm 1$ & $61 \pm 2$ & $58 \pm 2$ \\
2 & $25 \pm 1$ & $38 \pm 1$ & $59 \pm 2$ & $54 \pm 2$ \\
$3 *$ & $22 \pm 1$ & $60 \pm 2$ & $71 \pm 2$ & $76 \pm 2$ \\
$*$ *xygen poisoned & & &
\end{tabular}

\subsection{Measurement method}




\subsubsection{Elastomer thickness}

The true thickness of the elastomer films was determined by applying the elastomer to a glass plate and measuring the distance from this to the edge of the elastomer. The measurement was done on a Leica DMLB microscope with a 2.0 Thor lab USB 2.0 Digital Camera. This method has previously been proven reliable upon comparison with weighing large cut-outs of films with pre-specified areas.

\subsubsection{Dielectric breakdown strength}

Electrical breakdown strength measurements of the silicone elastomer were performed on an in-house-built machine adhering to international standards IEC 60243-1 (1998) and IEC 60243-2 (2001). The silicone film was placed between two hemispherical electrodes such that the electrodes touched the elastomer surfaces on both sides, with a small indentation ensuring contact. The distances between the two hemispherical electrodes were noted for each measurement, and the voltage was then increased by $0.1 \mathrm{kV} / \mathrm{s}$ increments until a short circuit occurred. Each elastomer film was tested 12 times.

\subsubsection{Tensile stress-strain}

The stress strain relationship of the silicone elastomer was measured by an ARES-G2 rheometer with an SER2 geometry. The measurements were performed with $6 \mathrm{~mm}$-wide samples of the silicone elastomer, which were attached horizontally on the SER2 module, in order to avoid askew stretching of the sample, which could have resulted in misleading results.

The stretch on the elastomer took place in the coils rotating in opposite directions, causing a steady Hencky strain rate of $0.01 \mathrm{~s}^{-1}$.

\section{Discussion and results}


The silicone elastomer LR3043/50, which contains extremely viscous elastomer premixes, was chosen for the investigations because it has excellent properties for dielectric elastomer uses, but at the same time it is extremely difficult to process without using significant amounts of solvents, thus making it very sensitive to correct processing.

Three batches of the elastomer were prepared by the same procedure but on different days. The first three films from each batch resulted from speed-mixing the elastomer premixes, with the third batch being exposed to oxygen for two weeks to cause some degradation of the silane crosslinker over time, and thus lower the degree of crosslinking in the final elastomer film. The presence of oxygen leads to oxidation of the $\mathrm{Si}-\mathrm{H}$ group which turns into $\mathrm{Si}-\mathrm{OH}$, and the $\mathrm{Si}-\mathrm{OH}$ group is not capable of crosslinking at the given conditions. Therefore crosslinker functionality is lost and the film must become inherently softer. The three films from speed-mixing were coated consecutively with knife gaps of 50, 100 and $150 \mu \mathrm{m}$, respectively. The fourth film in each batch was prepared by simple hand-mixing, i.e. not adequate mixing, and coated with a knife gap of $100 \mu \mathrm{m}$. As a result, batches 1 and 2 should yield the same results within experimental error, except for sample 4, where reproducibility was not expected due to the hand-mixing procedure. Batch 3 should have the characteristics of an insufficiently crosslinked elastomer compared to the reference elastomers in batches 1 and 2.

In Table 2, the mechanical properties of the various films are listed. The Young's moduli, as functions of film thickness, are also shown in Figure 3. From the achieved results it is clear that oxygen poisoning has by far the largest influence on mechanical properties, whereas improper mixing is not as easily detectable. However, what is evident from the table is that batches 1 and 2 compare well mechanically, whereas batch 3 has a significantly lower Young's modulus and tensile strength, thereby indicating a much lower degree of crosslinking, which will directly influence the electrical properties. ${ }^{[13]}$ Also it can be seen that 
all properly mixed samples show a slight decrease of Young's modulus with increased thickness. This indicates that the surfaces of the films cure better than the bulk. This is commonly known for condensation curing silicone elastomers but for addition curing elastomers, such as the one investigated within this study, this is not generally known and usually the Young's modulus is regarded independent of thickness.

Table 2. Mechanical properties of the films from the three batches. All films are speed-mixed except for the fourth in each batch (marked with "-h" to indicate the hand-mixing).

\begin{tabular}{llllll} 
Batch No. & $\begin{array}{l}\text { Sample } \\
\text { No. }\end{array}$ & $\begin{array}{l}\text { Thickness } \\
{[\mu \mathrm{m}]}\end{array}$ & $\begin{array}{l}\text { Y5\% } \\
{[\mathrm{MPa}]}\end{array}$ & $\begin{array}{l}\text { Tensile strength } \\
{[\mathrm{MPa}]}\end{array}$ & $\begin{array}{l}\text { Elongation at break } \\
(\%)\end{array}$ \\
\hline 1 & 1 & $31 \pm 1$ & $3.8 \pm 0.2$ & $14.6 \pm 0.3$ & $599 \pm 17$ \\
& 4 & $46 \pm 1$ & $3.6 \pm 0.1$ & $15.5 \pm 0.4$ & $624 \pm 21$ \\
& 3 & $61 \pm 2$ & $3.6 \pm 0.2$ & $15.6 \pm 0.3$ & $692 \pm 29$ \\
& $4-\mathrm{h}$ & $58 \pm 2$ & $5.1 \pm 0.2$ & $13.8 \pm 0.5$ & $589 \pm 34$ \\
\hline 2 & 1 & $25 \pm 1$ & $3.9 \pm 0.2$ & $13.6 \pm 0.4$ & $542 \pm 28$ \\
& 2 & $38 \pm 1$ & $3.7 \pm 0.1$ & $14.4 \pm 0.5$ & $575 \pm 26$ \\
& 3 & $59 \pm 2$ & $3.4 \pm 0.2$ & $14.6 \pm 0.5$ & $624 \pm 34$ \\
& $4-\mathrm{h}$ & $54 \pm 2$ & $4.2 \pm 0.2$ & $11.0 \pm 0.6$ & $493 \pm 39$ \\
\hline & 1 & $22 \pm 1$ & $2.4 \pm 0.2$ & $12.0 \pm 0.5$ & $565 \pm 25$ \\
& 2 & $60 \pm 2$ & $1.4 \pm 0.1$ & $6.7 \pm 0.2$ & $619 \pm 32$ \\
& 3 & $71 \pm 2$ & $1.4 \pm 0.1$ & $5.3 \pm 0.2$ & $488 \pm 38$ \\
& $4-\mathrm{h}$ & $76 \pm 2$ & $1.3 \pm 0.1$ & $6.7 \pm 0.4$ & $604 \pm 45$ \\
\hline
\end{tabular}

*Oxygen poisoned 


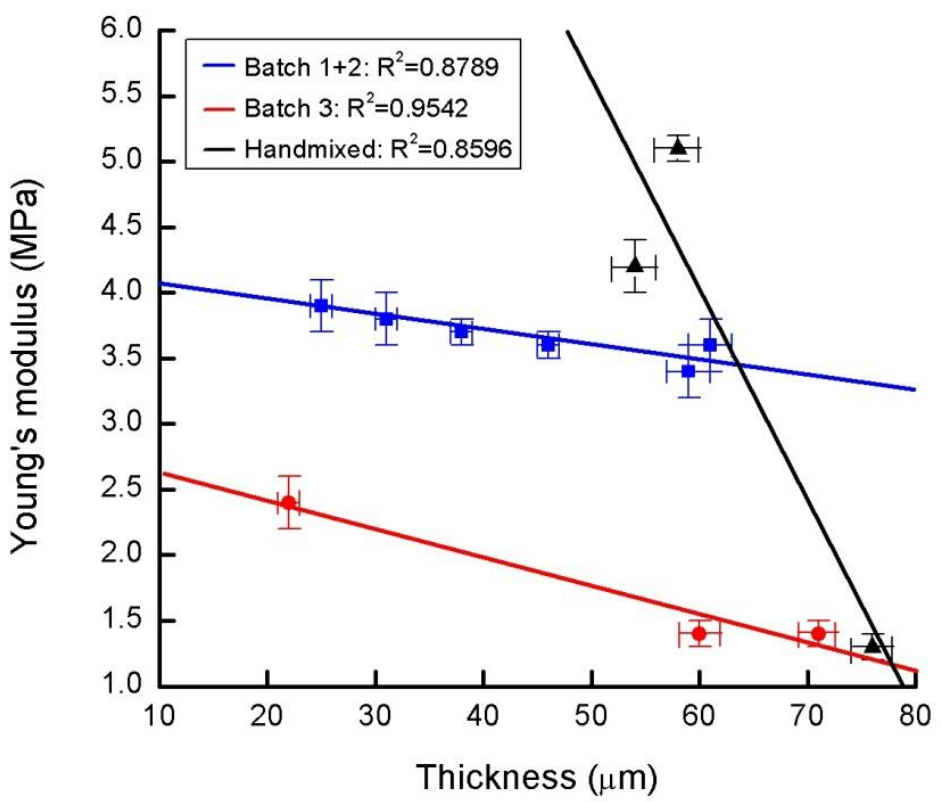

Fig.3. Young's moduli as functions of film thickness for the three different types of films (batches 1 and 2 being considered as ideal films, batch 3 with less crosslinking and handmixed samples being improperly processed).

With respect to electrical characterisation, voltage ramps were introduced to the various films until breakdown took place, as illustrated in Figure 4, from which it is apparent that the electrical breakdown gives rise to visible pinholes. The electrical breakdown patterns followed typical breakdowns for silicone elastomers, ${ }^{[27]}$ and for each elastomer sample 12 local electrical breakdown strengths were recorded. The electrical breakdown strength results in terms of the fitted Weibull parameters are illustrated in Table 3. 
Table 3. Weibull parameters and $R^{2}$ for the probability plot of the films from the three batches.

\begin{tabular}{llllll} 
Batch No. & Sample No. & $\begin{array}{l}\text { Thickness } \\
{[\mu \mathrm{m}]}\end{array}$ & $\beta$ & $\eta[\mathrm{V} / \mu \mathrm{m}]$ & $\mathrm{R}^{2}$ \\
\hline 1 & 1 & $31 \pm 1$ & 20.8 & 198 & 0.93 \\
& 2 & $46 \pm 1$ & 21.8 & 184 & 0.94 \\
& 3 & $61 \pm 2$ & 43.1 & 177 & 0.98 \\
& $4-\mathrm{h}$ & $58 \pm 2$ & 13.2 & 171 & 0.76 \\
\hline 2 & 1 & $25 \pm 1$ & 12.9 & 211 & 0.90 \\
& 2 & $38 \pm 1$ & 23.4 & 190 & 0.91 \\
& 3 & $59 \pm 2$ & 39.8 & 181 & 0.93 \\
& $4-\mathrm{h}$ & $54 \pm 2$ & 6.68 & 162 & 0.86 \\
& 1 & $22 \pm 1$ & 23.2 & 89.5 & 0.90 \\
& 2 & $60 \pm 2$ & 23.4 & 87.4 & 0.86 \\
& 3 & $71 \pm 2$ & 9.96 & 94.1 & 0.96 \\
& $4-\mathrm{h}$ & $76 \pm 2$ & 7.12 & 98.8 & 0.95 \\
\hline
\end{tabular}

*Oxygen-poisoned

In Figure 5, the probability density function of the electrical breakdowns for batch 2 (as a representative batch) is presented. Probability density functions of all samples can be seen in ESI. The thinnest film shows the largest scale parameter, $(\eta)$, which is representative of the highest electrical breakdown strength, though it also shows a relatively large width of 
distribution $(\beta)$, due to the relatively low value of $\beta$. The distribution thins significantly in line with increasing thickness, thus indicating more homogeneous breakdown patterns, but the electrical breakdown strength drops - the reason for which will be discussed subsequently. For the hand-mixed samples, the broadest distributions of all are achieved, thereby indicating a low degree of homogeneity together with low electrical breakdown strength values.

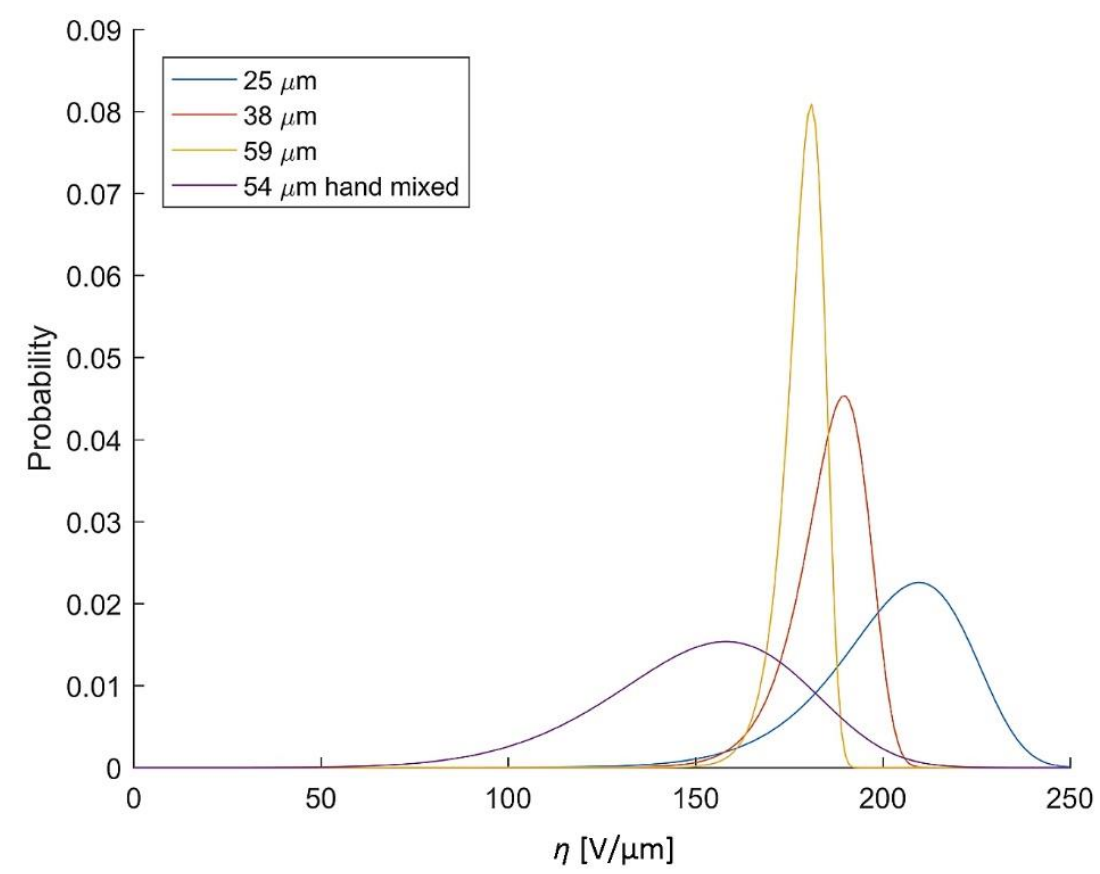

Fig. 5. Probability density functions for the four films from batch 2 indicating how the

Weibull distribution changes with film thickness. The actual film thicknesses are shown in the legend.

In Figure 6, a comparison across the batches on films targeted to be of the same thickness, and their respective probability density functions, is shown for the thickest sample (sample 3). Noticeablely, batch 3 falls significantly short of the two others with respect to both $\eta$ and $\beta$, which agrees well with the significant degree of crosslinking discussed previously. 


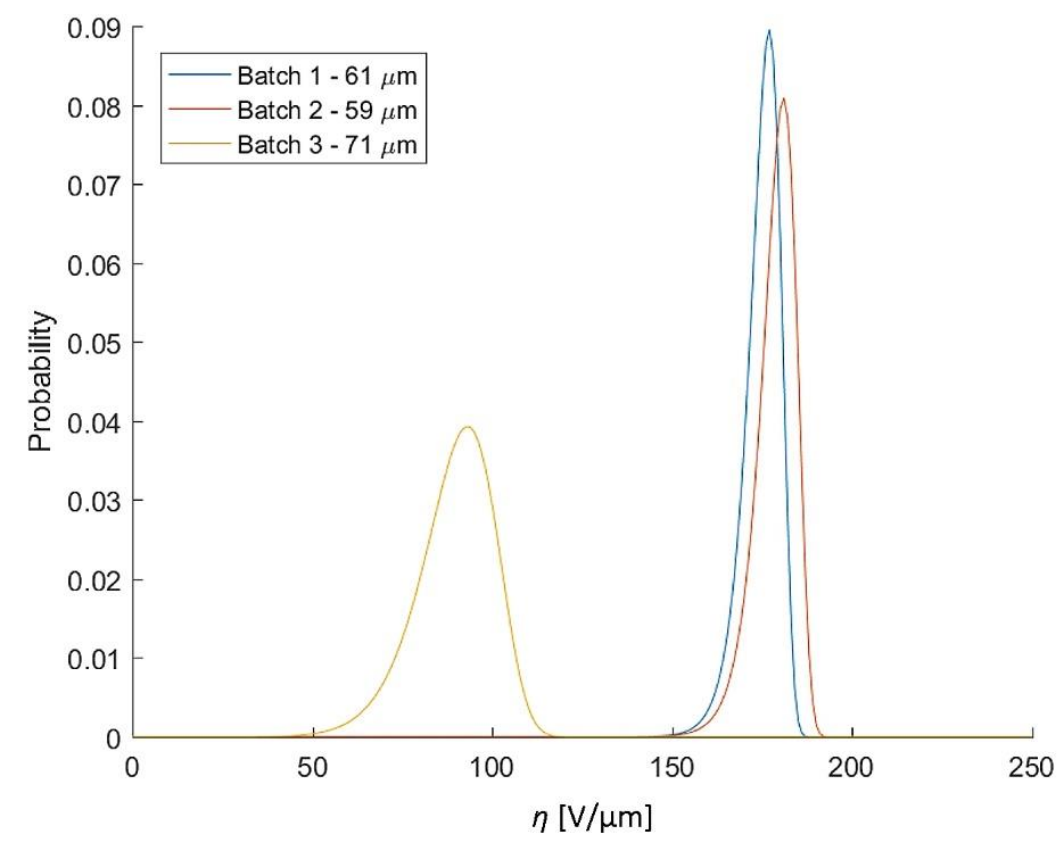

Fig. 6. Comparison of the thickest speed-mixed samples from batches 1-3. Batches 1 and 2 show excellent agreement, as expected, whereas batch 3 with the degraded crosslinker shows both significantly lower electrical breakdown strength $(\eta)$ and wider distribution (lower Weibull slope, $\beta)$.

Gate oxides in transistors have seen extensive modelling of dielectric properties. These gate oxides are composed of thin oxide films, in which the electrical breakdown has been modelled as a percolation of defects introduced by small currents passing through the material, shown as discrete units which are either conductive or non-conductive. ${ }^{[28-31]}$ These models have led to a theoretical estimation that agrees well with our experimental finding, namely that $\beta$ increases with sample thickness. ${ }^{[29,30]}$ In thin oxide films, the breakdown is induced by stress provoked by low voltages, but the thickness is correspondingly smaller, ${ }^{[31]}$ so the field strengths are of the same magnitude as in elastomer electrical breakdown strength measurements. It can therefore be hypothesised that electrical breakdown in silicone elastomers can be modelled by the same percolation theories used in the previously discussed theories. ${ }^{[28,30]}$ Furthermore, the increase in shape parameter $(\beta)$ in line with thickness can be 
explained by the fact that more defects are needed to reach the percolation threshold, thereby reducing the likelihood of a conducting path being formed. ${ }^{[29]}$

If a probability, $p$, that a given bond or connection is conductive is defined, then the percolation threshold for overall conduction has been shown to follow the following relation for specific dimensions and bond configurations ${ }^{[32]}$ :

$$
p_{c}(h)-p_{c 3} \sim h^{-5 / 4}
$$

where $h$ is the thickness of the sample and $p_{c 3}=0.278$ is a critical percolation threshold in a bulk $3 \mathrm{D}$ array for fully amorphous distributions of bonds/interconnections (i.e. $h \rightarrow \infty) .{ }^{[32]}$ Equation 6 holds only for $\frac{h}{L}>0.1$, where $L$ is the width of the specimen (in our case $L$ will be representative of the diameter of the contact area, which is significantly larger than the thickness of the elastomer). The above expression indicates that as the thickness of the elastomer increases, the percolation threshold decreases, i.e. it becomes relatively easier to create a conducting path through the thickness (at any given probability $p$ ), and thus the electrical breakdown strength is lower for thicker samples.

If we assume that electrical breakdown strength is reached when the bonds percolate, then we can write:

$$
\eta \sim p_{c}(h) \sim h^{-5 / 4}
$$

This correlation is plotted in Figure 7 versus thickness for the two identical batches 1 and 2 . It is obvious that there is an excellent fit $\left(\mathrm{R}^{2}=0.996\right)$ of the proposed theory to our experimental data. Furthermore in Figure 7 a linear fit of $\beta$ versus thickness has been made. It is not believed that this relationship is universal but it gives a clear indication that decreased film thickness comes at a cost with respect to the homogeneity of breakdowns for blade coated films. 

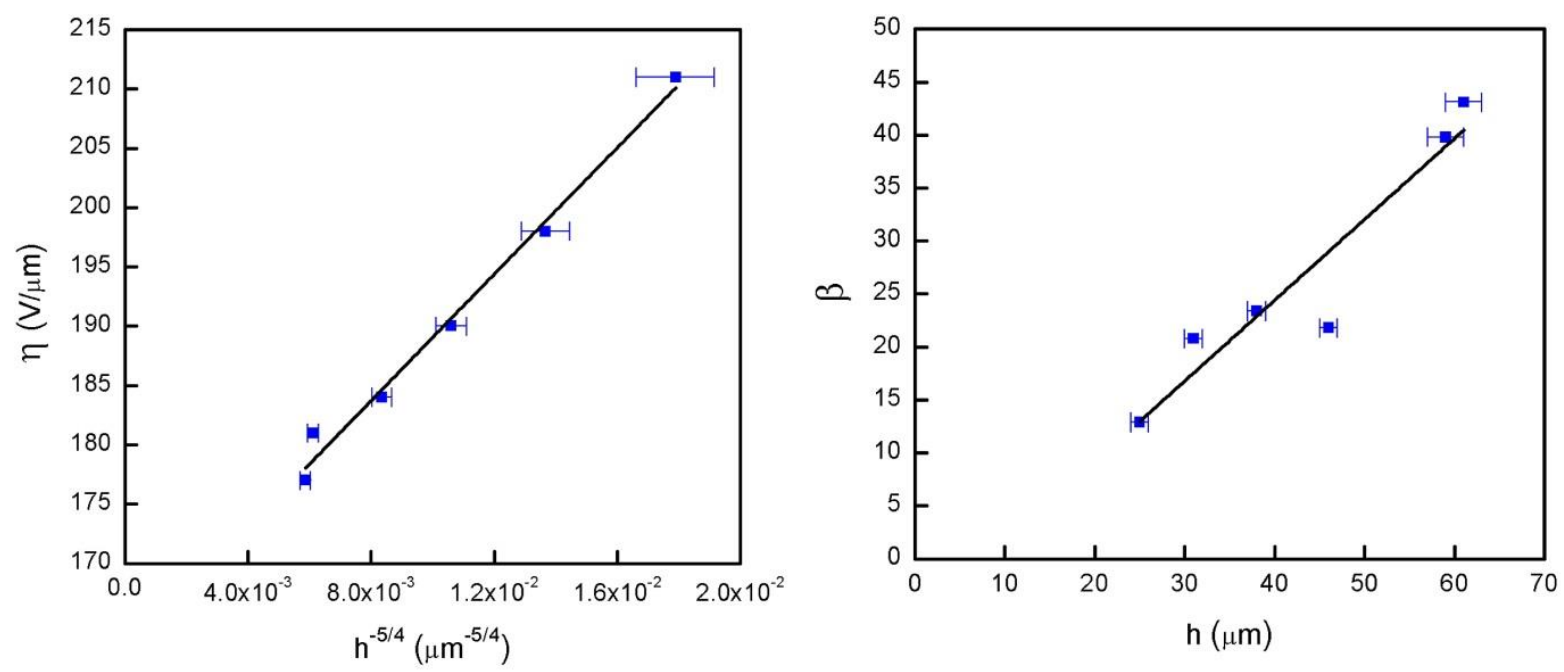

Fig. 7. Scale and shape parameters for speed-mixed and non-degraded batches (1 and 2)

versus elastomer thickness. The scale parameter is fitted by means of the prediction from the percolation theory, whereas the shape parameter is just fitted with a linear relationship.

For the hand-mixed samples, there is no correlation between $\beta$ and thickness or $\eta$ and thickness, respectively, as seen for the speed-mixed samples. The sheer difference between the speed-mixed and hand-mixed samples is that homogenisation is uncontrolled in hand- 
mixing, and almost full homogenisation is obtained with speed-mixing. ${ }^{[33]}$ Therefore, it is clear that both $\beta$ and $\eta$ are dependent on homogeneity. It can also be seen that the speedmixed samples have a higher $\beta$ value than the hand-mixed samples, which again confirms that $\beta$ increases with homogeneity.

\section{Conclusions}

It was established herein that electrical breakdown strength determinations provide direct information about both elastomer quality and processing, though this distinction cannot be performed by analysing mechanical properties. It was discussed that by improperly mixing very viscous premixes, so-called "heterogeneous networks" can be achieved that exhibit complex mechanical behaviours due to inhomogeneities in crosslinking density working as reinforcing domains. Therefore, no distinct definition of elastomer mixing could be found from the Young's modulus determinations. However, from electrical breakdown strength determinations, these inhomogeneities were apparent through changes in electrical breakdown strength $(\eta)$ as well as in the width of the probability density function $(\beta)$. Poisoning of the crosslinker (i.e. chemical changes) could be detected by both mechanical and electrical characterisation.

This is one step towards detecting film quality and implicitly elastomer mixing, albeit in the current state not online. However, detection could be implemented by testing the outer area of the dielectric elastomer films in an online manner, ideally by utilising self-healing elastomers such as those proposed in recent work. ${ }^{[34-36]}$

\section{Acknowledgements}

Harald Silau, Nicolai Bogø Stabell and Frederik Riddersholm Petersen contributed equally to the work. The authors wish to express thanks to Ramona Valentina Mateiu (Hempel 
Foundation Coatings Science and Technology Center, DTU) for help with the SEM investigation. Anne Ladegaard Skov and Liyun Yu appreciate the Danish Research Council's financial support for the project.

Received: ((will be filled in by the editorial staff))

Revised: ((will be filled in by the editorial staff))

Published online: ((will be filled in by the editorial staff))

\section{References}

[1] F. B. Madsen, A. E. Daugaard, S. Hvilsted, A. L. Skov, Macromolecular Rapid Communications 2016, 37, 378.

[2] P. Brochu, Q. Pei, Macromolecular Rapid Communications 2010, 31, 10.

[3] S. Ito, S. M. Zakeeruddin, R. Humphry-Baker, P. Liska, R. Charvet, P. Comte, M. K. Nazeeruddin, P. Péchy, M. Takata, H. Miura, S. Uchida, M. Grätzel, Advanced Materials 2006, $18,1202$.

[4] A. Armin, M. Hambsch, P. Wolfer, H. Jin, J. Li, Z. Shi, P. L. Burn, P. Meredith, Advanced Energy Materials 2015, 5, 1401221.

[5] F. Carpi, I. Anderson, S. Bauer, G. Frediani, G. Gallone, M. Gei, C. Graaf, C. JeanMistral, W. Kaal, G. Kofod, M. Kollosche, R. Kornbluh, B. Lassen, M. Matysek, S. Michel, S. Nowak, B. O’Brien, Q. Pei, R. Pelrine, B. Rechenbach, S. Rosset, H. Shea, Smart Materials and Structures 2015, 24, 105025.

[6] D. McCoul, S. Rosset, S. Schlatter, H. Shea, Smart Materials and Structures 2017, 26, 125022.

[7] S. Rosset, H. R. Shea, Applied Physics Reviews 2016, 3, 031105.

[8] T. Töpper, B. Osmani, B. Müller, Microelectronic Engineering 2018, 194, 1.

[9] R. Pelrine, R. Kornbluh, Q. Pei, J. Joseph, Science 2000, 287, 836.

[10] X. Zhao, Z. Suo, Physical Review Letters 2010, 104, 178302. 
[11] G. Zurlo, M. Destrade, D. DeTommasi, G. Puglisi, Physical Review Letters 2017, $118,078001$.

[12] J. Chin, E. S. Boek, P. V. Coveney, Philosophical Transactions of the Royal Society A-mathematical Physical and Engineering Sciences 2002, 360, 547.

[13] A. L. Skov, L. Yu, Adv. Eng. Mater. DOI: 10.1002/adem.201700762.

[14] F. B. Madsen, A. E. Daugaard, C. Fleury, S. Hvilsted, A. L. Skov, RSC Advances 2014, 4, 6939.

[15] A. L. Larsen, P. Sommer-Larsen, O. Hassager, Proceedings of SPIE 2004, 5385, 108.

[16] F. Di Lorenzo, S. Seiffert, Polymer Chemistry 2015, 6, 5515.

[17] O. Friedrich, D. Schneidereit, Y. A. Nikolaev, V. Nikolova-Krstevski, S. Schürmann, A. Wirth-Hücking, A. L. Merten, D. Fatkin, B. Martinac, Progress in Biophysics and Molecular Biology 2017, 130, 170.

[18] F. B. Madsen, S. B. Zakaria, L. Yu, A. L. Skov, Advanced Engineering Materials 2016, 18,1154 .

[19] M. Kollosche, G. Kofod, Applied Physics Letters 2010, 96, 071904.

[20] S. Zakaria, P. H. F. Morshuis, M. Y. Benslimane, L. Yu, A. L. Skov, Smart Materials and Structures 2015, 24, 055009.

[21] H. Rinne, The Weibull distribution: a handbook, Chapman and Hall/CRC, New York, United States, 2008.

[22] S. B. Zakaria, F. B. Madsen, A. L. Skov, Polymer-Plastics Technology and Engineering 2017, 56, 83.

[23] A. H. A Razak, A. L. Skov, RSC Advances 2017, 7, 468.

[24] H. Stoyanov, P. Brochu, X. Niu, C. Lai, S. Yun, Q. Pei, RSC Advances 2013, 3, 2272. 
[25] S. Michel, B. T. T. Chu, S. Grimm, F. A. Nüesch, A. Borgschulte, D. M. Opris, Journal of Materials Chemistry 2012, 22, 20736.

[26] M. A. Brook, H.-U. Saier, J. Schnabel, K. Town, M. Maloney, Industrial \& Engineering Chemistry Research 2007, 46, 8796.

[27] L. Yu, F. B. Madsen, A. L. Skov, International Journal of Smart and Nano Materials. DOI: 10.1080/19475411.2017.1376358.

[28] J. Sune, IEEE Electron Device Letters 2001, 22, 296.

[29] E. Y. Wu, J. Sune, W. Lai, IEEE Transactions on Electron Devices 2002, 49, 2141.

[30] J. H. Stathis, Journal of Applied Physics 1999, 86, 5757.

[31] J. H. Stathis, D. J. DiMaria, International Electron Devices Meeting 1998, 167.

[32] L. Zekri, A. Kaiss, J.-P. Clerc, B. Porterie, N. Zekri, Physics Letters A 2011, 375, 346.

[33] S. S. Hassouneh, L. Yu, A. L. Skov, A. E. Daugaard, Journal of Applied Polymer Science 2017, 134, 44767.

[34] F. B. Madsen, L. Yu, A. L. Skov, ACS Macro Letters 2016, 5, 1196.

[35] S. J. Dünki, Y. S. Ko, F. A. Nüesch, D. M. Opris, Advanced Functional Materials 2015, 25, 2467.

[36] L. Yu, F. B. Madsen, S. Hvilsted, A. L. Skov, RSC Advances 2015, 5, 49739. 
Electrical breakdown strength characterisation is shown as being able to detect chemical changes in the elastomer premixes, as well as improper mixing of the premixes, in a very efficient manner. Weibull analysis of the individual local breakdown strengths further provides information on the resulting elastomer films. The obtained data are also shown to agree very well with the percolation theories from gate oxides in transistors, where each interconnection is modelled as either conducting or non-conducting. Thereby, a scaling law for electrical breakdown strength as a function of film thickness can be derived.

Harald Silau, Nicolai Bog $\varnothing$ Stabell, Frederik Riddersholm Petersen, Martin Pham, Liyun Yu, Anne Ladegaard Skov*

Weibull analysis of electrical breakdown strength as an effective means of evaluating elastomer thin film quality

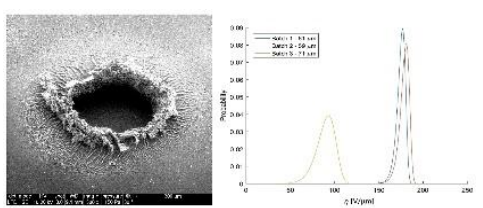

